\title{
Estimating brain volume loss after radiation therapy in children treated for posterior fossa tumors (Corpus callosum and whole brain volume changes following radiotherapy in children)
}

\author{
Elwira Szychot ${ }^{1, A-D, F}$, Kiran K. Seunarine ${ }^{2, A, C, F}$, Carlos Andrés Robles ${ }^{3, B, C, F}$, Henry Mandeville, ${ }^{1, A, E}$, \\ Kshitij Mankad ${ }^{4, C, E}$, Christopher Clark ${ }^{2, E}$, Jarosław Peregud-Pogorzelski ${ }^{5, C, E}$, Nandita deSouza ${ }^{6, A, C, E, F}$ \\ ${ }^{1}$ The Oak Centre for Children and Young People, Royal Marsden Hospital, Sutton, London, UK \\ 2 Developmental Imaging and Biophysics Section, Institute of Child Health, University College London, UK \\ ${ }^{3}$ Department of Radiology, Exequiel Gonzalez Cortes Children's Hospital, Santiago, Chile \\ ${ }^{4}$ Department of Radiology, Great Ormond Street Hospital for Children NHS Foundation Trust, London, UK \\ ${ }^{5}$ Department of Pediatrics and Pediatric Oncology, Pomeranian Medical University, Szczecin, Poland \\ ${ }^{6}$ CRUK Imaging Centre at The Institute of Cancer Research, Sutton, London, UK \\ A - research concept and design; B - collection and/or assembly of data; $C$ - data analysis and interpretation; \\ $D$ - writing the article; $E$ - critical revision of the article; $F$ - final approval of the article
}

\section{Address for correspondence \\ Elwira Szychot \\ E-mail: e.szychot@nhs.net}

\section{Funding sources}

None declared

\section{Conflict of interest}

None declared

\section{Acknowledgements}

We acknowledge the support from the NIHR Biomedical Research Centre at the RMH/ICR.

\section{Received on April 8, 2019}

Reviewed on April 15, 2019

Accepted on November 26, 2019

Published online on March 26, 2020

Cite as

Szychot E, Seunarine KK, Robles CA, et al. Estimating brain volume loss after radiation therapy in children treated for posterior fossa tumors (Corpus callosum and whole brain volume changes following radiotherapy in children). Adv Clin Exp Med. 2020;29(3):331-337.

doi:10.17219/acem/114827

DOI

10.17219/acem/114827

\section{Copyright}

Copyright by Author(s)

This is an article distributed under the terms of the

Creative Commons Attribution 3.0 Unported (CC BY 3.0)

(https://creativecommons.org/licenses/by/3.0/)

\section{Abstract}

Background. More than half of pediatric tumors of central nervous system (CNS) primarily originate in the posterior fossa and are conventionally treated with radiation therapy (RT).

Objectives. The objective of this study was to establish whether corpus callosum volumes (CCV) and whole brain volumes (WBV) are correlated and to determine the impact of whole-brain low-vs high-dose RT on brain parenchymal volume loss as assessed using each technique.

Material and methods. Of the 30 identified children (6-12 years) with newly diagnosed posterior fossa tumors treated with cranial RT, including focal and whole-brain RT, suitable imaging was obtained for 23. Radiotherapy regimens were the following: no whole-brain RT (Group 1, $n=7$ ), low-dose whole-brain RT ( $<30$ Gy, Group 2, n =9) and high-dose whole-brain RT ( $>30$ Gy, Group 3, n=7) in addition to focal boost. Magnetic resonance images (MRIs) were analyzed at baseline and follow-up (median 14 months). The CCVs were manually segmented on midline sagittal slice $(n=23)$, while WBVs were segmented semi-automatically using Freesurfer $(n=15)$. This was done twice (6-month interval) for all baseline CCV measurements and 5 randomly selected WBV measurements to establish measurement reproducibility. Correlations between CCV and WBV were investigated and percentage of children demonstrating reduction in $C C V$ or WBV noted.

Results. Correlation between baseline CCV and WBV was not significant ( $p=0.37$ ). Measurement reproducibility was from $6 \%$ to $-9 \%$ for CCV and from $4.8 \%$ to $-1.2 \%$ for WBV. Among the children studied, $30.4 \%$ (7/23) had $>9 \%$ reduction in CCV at follow-up, while 33.3\% (5/15) had $>1.2 \%$ reduction in WBV. Five of 7 patients with CCV loss were not picked up by WBV measurements. Similarly, 3 of 5 patients with WBV loss were not picked up by CCV measurements.

Conclusions. The CCV and the WBV are unrelated and may indicate different brain parenchymal losses following RT. Up to a third of posterior fossa tumors treated with RT have measurable CCV or WBV loss; incidence was equivalent in low- vs high-dose whole-brain RT.

Key words: posterior fossa tumor, radiotherapy, brain volume 


\section{Introduction}

More than half of pediatric tumors of the central nervous system (CNS) primarily originate in the posterior fossa and are conventionally treated with radiation therapy (RT). ${ }^{1}$ Such therapy is extremely effective and has led to an increase in long-term survivorship. However, this treatment induces neurotoxicity, manifesting as various forms of motor and cognitive long-term impairment, and is problematic in individuals in whom there is a long life expectancy: quality of life in children surviving treatment of CNS tumors is recognized as being of vital importance. ${ }^{2,3}$ Neurobehavioral morbidity is a late outcome measure, with limited management options, which is why early objective indicators of likely motor or cognitive deficit are increasingly sought in order to implement appropriate management strategies early. ${ }^{3}$

Magnetic resonance imaging (MRI) has revolutionized anatomic assessments of the normal and diseased brain and has enabled characterization and monitoring of structural changes within the brain resulting from RT. Various degrees of parenchymal volume loss and generalized white matter signal changes have been reported in children treated for medulloblastoma (MDL). ${ }^{4-7}$ Most studies used a fully automated hybrid neural network segmentation as well as a classification method to quantitatively derive volumes of brain parenchyma from these images. ${ }^{4,7,8} \mathrm{Vol}$ umes of the corpus callosum (CC) have also been advocated as a surrogate to quantify volumes of neuroparenchyma, because the nearly 180 million myelinated axons within this white matter commissure make it susceptible to radiation-induced damage. ${ }^{5,9}$ Nevertheless, a correlation between $\mathrm{CC}$ volume (CCV) and whole brain volume (WBV) has not been demonstrated. The purpose of this study, therefore, was to establish whether CCVs and WBVs are correlated and to determine the impact of low-vs highdose RT on brain parenchymal volume loss as assessed using each technique.

\section{Material and methods}

\section{Patients}

Approval for this study was obtained from the Institutional Review Board. Informed consent from the parents was waived.

We searched the pediatric oncology database of the Royal Marsden Hospital (Sutton, London, UK) regarding a period from 2000 to 2013 and identified 30 children between the age of 6 and 12 with newly diagnosed posterior fossa tumors, who were treated with cranial RT and in whom baseline (pre-RT) and follow-up (at least 6 months after treatment) MRI scans were available. Radiotherapy regimens were either focal to the posterior fossa only or included whole-brain RT at low (<30 Gy) or high ( $>30$ Gy) dose.
Children below the age of 6 and those above the age of 12 were excluded from the study to ensure the similar age of our cohort. Other exclusion criteria were the following: 1) radiological evidence of surgery-related intracranial bleeding (excluding asymptomatic, resolving hemorrhagic changes associated with recent surgery and the presence of punctate hemorrhage in the tumor), 2) any disease or condition that disabled compliance according to the appropriate radiation regimen, 3) prior diagnosis of malignancy and disease during the last imaging followup, 4) previous cranial irradiation, 5) prior systematic anticancer therapy, and 6) images with artefact that precluded brain parenchymal volume measurements.

\section{MRI evaluation}

Selection of the MRI scans for the $2^{\text {nd }}$ timepoint was based on the completeness of image data available. Selected images where anonymized and placed in a Cancer Research UK (CRUK) Cancer Imaging Centre repository.

All images were visually inspected for quality. T2weighted images, pre- and post-contrast T1-weighted images and fluid attenuated inversion recovery and diffusion weighted imaging/apparent diffusion coefficient maps were reviewed for each patient. The MRI scans were retrospectively and quantitatively analyzed by a specialist pediatric neuroradiologist and a biomedical scientist. Both readers were blind to group membership and the timepoint when the scans were taken.

\section{Corpus callosum volume measurement}

The anonymized images were opened in OsiriX imaging Software (Pixmeo, Geneva, Switzerland) and a region of interest (ROI) was manually drawn on the mid-sagittal slice of T1-W images. Manual segmentations of CC were retrospectively performed by a pediatric radiologist with 5 years of experience in pediatric brain MRI. The CCV was calculated using the FMRIB Software Library's (FSL) stats command (https://fsl.fmrib.ox.ac.uk/fsl/fslwiki/FSL). To account for differences in acquisition, the volume of ROI was normalized by slice thickness. ${ }^{10}$ Six months after the initial ROIs were drawn, a $2^{\text {nd }}$ set of CC ROIs were manually drawn by the original observer on each midsagittal images to establish repeatability of the method.

\section{Brain volume measurement}

A mask of each patient's brain was generated using the autorecon1 command in FreeSurfer v. 5.3 (https://surfer. nmr.mgh.harvard.edu). ${ }^{11}$ Each brain mask was then manually corrected by a trained biomedical scientist with 5 years of experience in pediatric neuroimaging. The brain masks were transformed into halfway space and further masked to ensure that only regions within the field of view at both timepoints were included in the volume analysis. Whole 
brain volumes were calculated by summing the voxels within the brain mask using FMRIB Software Library's (FSL) stats command (https://fsl.fmrib.ox.ac.uk/fsl/fslwiki/ FSL). The MRI examinations of 5 patients were randomly selected from the dataset and measurements repeated 6 months later to establish reproducibility of the method.

\section{Statistical analysis}

Statistical analysis was performed using Prism v. 7.0 software (GraphPad Software, San Diego, USA). Descriptive statistics were used to summarize the measurements. As the data were non-parametric, a Spearman's rank correlation tested the association between $\mathrm{CCV}$ and WBV.

The intra-observer repeatability for each of the measurement techniques - absolute CCV or WBV - were assessed using a Bland-Altman method to calculate $95 \%$ confidence intervals (95\% CIs) of the measurement. A p-value of less than 0.05 was considered significant. The percentage reduction in CCV and WBV beyond these 95\% CIs was assumed to represent real reductions beyond measurement variability. The number of patients in each group that exceeded the measurement reproducibility limits was noted, but small numbers precluded meaningful statistical comparisons.

\section{Results}

\section{Patients' characteristics}

Images of 23 children with complete data were included in final analyses. The cohort consisted of 17 males and 6 females with a median age of 10 years (range: $6-12$ years) at the time of diagnosis. Out of 23 children, 7 were diagnosed with high-risk MDL (MDL HR), 9 with standard-risk MDL (MDL SR), 3 with ependymoma, 3 with low-grade glioma (LGG), and 1 with atypical teratoid rhabdoid tumor (ATRT). Children diagnosed with MDL and ependymoma had received adjuvant treatment. Those with MDL had also received craniospinal irradiation with boost to the posterior fossa and chemotherapy, whereas patients diagnosed with gliomas had not (Table 1).

Of 23 children, 7 received focal RT to the posterior fossa only (group 1), 9 were treated additionally with wholebrain RT of 23.4 Gy in 13 fractions of 1.8 Gy (classified here as low-dose RT of $<30$ Gy - group 2), while the other 7 received $>30$ Gy to the whole brain and were considered high-dose patients ( 5 received whole-brain RT of $36 \mathrm{~Gy}$ in 20 daily fractions of 1.8 Gy, while 2 received 39 Gy in 30 fractions of 1.3 Gy to the posterior fossa - group 3).

\section{Intra-observer measurement variability}

Reproducibility estimates for the CCV ranged from 6\% to $-9 \%$ (Fig. 1A). The $95 \%$ CI for absolute CCV ranged from
Table 1. Patient characteristics: age, tumor type, radiation dose, time interval between the end of RT and the follow-up MRI. All patients underwent surgical resection prior to RT

\begin{tabular}{|c|c|c|c|c|}
\hline ID & $\begin{array}{c}\text { Age } \\
\text { [years] }\end{array}$ & DGN & $\begin{array}{c}\text { WB RT dose } \\
\text { [Gy] }\end{array}$ & $\begin{array}{l}\text { Time interval } \\
\text { [months] }\end{array}$ \\
\hline 1 & 10 & MDL HR & $>30$ & 6 \\
\hline 2 & 10 & MDL SR & $<30$ & 14 \\
\hline 3 & 9 & MDL SR & $<30$ & 73 \\
\hline 4 & 9 & LGG & 0 & 21 \\
\hline 5 & 11 & MDL HR & $>30$ & 9 \\
\hline 6 & 12 & EPND & 0 & 27 \\
\hline 7 & 9 & MDL SR & $<30$ & 48 \\
\hline 8 & 9 & MDL SR & $<30$ & 11 \\
\hline 9 & 10 & MDL SR & $<30$ & 60 \\
\hline 10 & 11 & MDL SR & $<30$ & 9 \\
\hline 11 & 7 & MDL HR & $>30$ & 12 \\
\hline 12 & 8 & ATRT & 0 & 10 \\
\hline 13 & 10 & MDL HR & $>30$ & 13 \\
\hline 14 & 10 & MDL SR & $<30$ & 60 \\
\hline 15 & 8 & LGG & 0 & 6 \\
\hline 16 & 6 & MDL HR & $>30$ & 38 \\
\hline 17 & 10 & MDL SR & $<30$ & 83 \\
\hline 18 & 8 & LGG & 0 & 96 \\
\hline 19 & 10 & EPND & 0 & 80 \\
\hline 20 & 11 & MDL SR & $<30$ & 74 \\
\hline 21 & 11 & MDL HR & $>30$ & 12 \\
\hline 22 & 11 & MDL HR & $>30$ & 10 \\
\hline 23 & 6 & EPND & 0 & 6 \\
\hline
\end{tabular}

RT - radiotherapy; MRI - magnetic resonance imaging; ID - patients anonymized number; DGN - diagnosis/tumor type; WB RT dose - RT dose to whole brain: 0 (only focal RT), <30 Gy or >30 Gy; time interval - time in months between the end of RT and the follow-up MRI; MDL HR - high-risk medulloblastoma; MDL SR - standard risk medulloblastoma; ATRT - atypical teratoid rhabdoid tumor; EPND - ependymoma; LGG - low-grade glioma.

$+0.48 \mathrm{~cm}^{3}$ to $-0.77 \mathrm{~cm}^{3}$. The WBV values had a smaller variability ranging from $4.8 \%$ to $-1.2 \%$ (Fig. 1B); absolute values ranged from $+5.5 \mathrm{~cm}^{3}$ to $-1.5 \mathrm{~cm}^{3}$.

\section{Comparison of corpus callosum and brain volume measurements as indicators of brain parenchymal loss}

Measurements at follow-up were performed in a range from 6 to 96 months after RT (median: 14 months, lower quartile (LQ): 10 months, upper quartile (UQ): 60 months).

Poor image quality made the derivation of the automated WBV segmentation error-prone, so that WBV measurements were obtainable in 15 out of 23 patients. Absolute $\mathrm{CCV}$ values for 23 children at both timepoints (baseline and follow-up), and WBV values in 15 children at those timepoints are summarized in Table 2. At baseline, CCV values ranged from $+4.4 \mathrm{~cm}^{3}$ to $-11.0 \mathrm{~cm}^{3}$ (median: $8.1 \mathrm{~cm}^{3}$ ), 
Table 2. Corpus callosum volume (CCV) and whole brain volume (WBV) measurements performed by the same observer twice at baseline and twice at follow-up (FU)

\begin{tabular}{|c|c|c|c|c|c|c|c|c|c|c|}
\hline ID & $\begin{array}{l}\text { Baseline CC } \\
\text { vol } 1\left[\mathrm{~cm}^{3}\right]\end{array}$ & $\begin{array}{c}\text { FU CC } \\
\text { vol } 1\left[\mathrm{~cm}^{3}\right]\end{array}$ & $\begin{array}{l}\text { Baseline CC } \\
\text { vol } 2\left[\mathrm{~cm}^{3}\right]\end{array}$ & $\begin{array}{c}\text { FU CC } \\
\text { vol } 2\left[\mathrm{~cm}^{3}\right]\end{array}$ & $\begin{array}{c}\text { Baseline } \\
\text { WBV } 1 \\
{\left[\mathrm{~cm}^{3}\right]}\end{array}$ & $\begin{array}{c}\text { FU WBV } 1 \\
{\left[\mathrm{~cm}^{3}\right]}\end{array}$ & $\begin{array}{c}\text { Baseline } \\
\text { WBV } 2 \\
{\left[\mathrm{~cm}^{3}\right]}\end{array}$ & $\begin{array}{c}\text { FU WBV } 2 \\
{\left[\mathrm{~mm}^{3}\right]}\end{array}$ & $\begin{array}{l}\text { Percentage } \\
\text { change CCV }\end{array}$ & $\begin{array}{l}\text { Percentage } \\
\text { change WBV }\end{array}$ \\
\hline 1 & 8.2 & 8.1 & 8.4 & 8.1 & & & & & -1.6 & \\
\hline 2 & 8.1 & 8.4 & 8.4 & 8.2 & 947.7 & 990.2 & 934.5 & 996.5 & 3.4 & 4.5 \\
\hline 3 & 9.0 & 7.9 & 9.3 & 7.5 & $1,119.4$ & $1,083.5$ & $1,087.7$ & $1,071.4$ & -13.6 & -3.2 \\
\hline 4 & 8.8 & 7.2 & 8.8 & 7.4 & & & & & -21.2 & \\
\hline 5 & 8.4 & 7.7 & 8.5 & 7.6 & & & & & -9.2 & \\
\hline 6 & 6.8 & 6.2 & 6.8 & 6.3 & & & & & -8.8 & \\
\hline 7 & 9.5 & 9.6 & 9.5 & 9.5 & $1,166.8$ & 1,186.0 & $1,129.1$ & $1,160.1$ & 1.0 & 1.6 \\
\hline 8 & 10.0 & 6.8 & 11.0 & 6.9 & $1,242.3$ & $1,256.4$ & & & -46.9 & 1.1 \\
\hline 9 & 10.9 & 8.5 & 11.2 & 8.5 & $1,308.1$ & $1,311.0$ & $1,283.5$ & $1,277.1$ & -29.2 & 0.2 \\
\hline 10 & 6.8 & 6.8 & 6.9 & 6.8 & & & & & 0.0 & \\
\hline 11 & 7.3 & 7.1 & 7.4 & 7.2 & & & & & -2.1 & \\
\hline 12 & 6.4 & 6.7 & 6.3 & 6.6 & & & & & 3.3 & \\
\hline 13 & 8.1 & 7.5 & 8.1 & 7.6 & 695.1 & 718.0 & & & -7.4 & 3.3 \\
\hline 14 & 7.4 & 8.3 & 8.6 & 8.3 & $1,304.9$ & $1,096.8$ & & & 10.8 & -15.9 \\
\hline 15 & 6.7 & 6.5 & 6.8 & 6.6 & $1,157.7$ & $1,160.6$ & $1,164.78$ & $1,159.4$ & -1.8 & 0.3 \\
\hline 16 & 6.2 & 5.3 & 6.2 & 5.2 & & & & & -17.0 & \\
\hline 17 & 7.0 & 7.7 & 7.1 & 7.7 & 1,319.2 & 1,331.0 & & & 9.5 & 0.9 \\
\hline 18 & 4.4 & 4 & 4.4 & 4.2 & $1,054.1$ & 977.3 & & & -9.7 & -7.3 \\
\hline 19 & 5.8 & 5.9 & 5.7 & 5.8 & $1,049.0$ & 1,059.7 & & & 0.7 & 1.0 \\
\hline 20 & 8.0 & 8.3 & 8.0 & 8.2 & $1,338.7$ & $1,400.7$ & & & 3.0 & 4.6 \\
\hline 21 & 6.9 & 7 & 6.8 & 7.1 & $1,144.4$ & $1,122.2$ & & & 1.1 & -1.9 \\
\hline
\end{tabular}

Baseline CC vol 1 - first measurement of CCV at diagnosis; FU CC vol 1 - first measurement of CCV at follow-up; Baseline CC vol 2 - repeated measurement of baseline CCV at diagnosis; FU CC vol 2 - repeated measurement of CCV at follow-up; Baseline WBV 1 - first measurement of the WBV at diagnosis; FU WBV 1 - first measurement of the WBV at follow-up; Baseline WBV 2 - repeated measurement of the WBV at diagnosis; FU WBV 2 - repeated measurement of the WBV at follow-up.
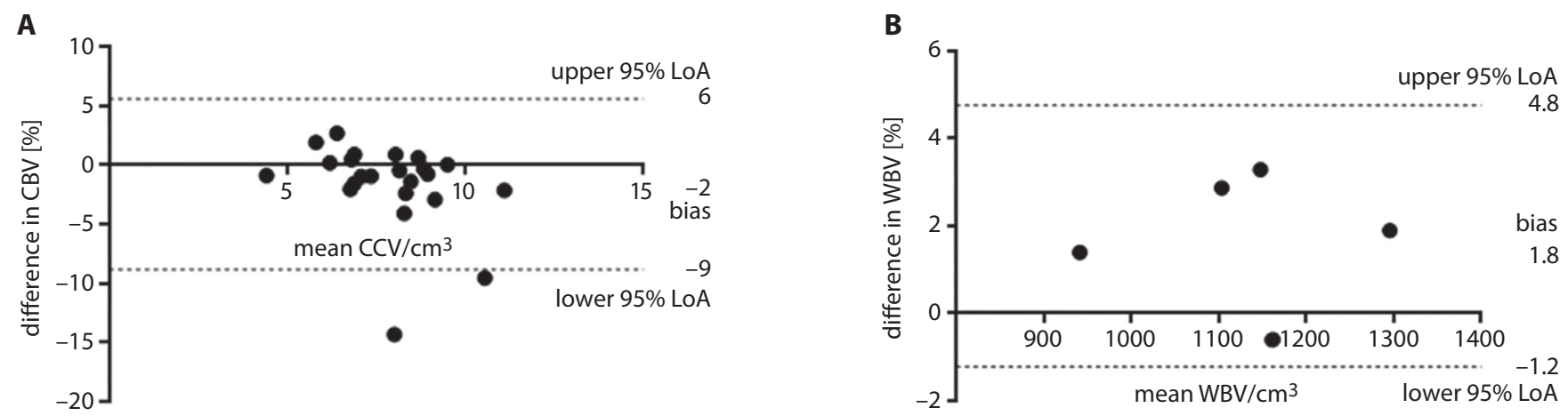

Fig. 1. A. Bland-Altman plots showing intra-observer reproducibility and upper and lower 95\% Cls (dotted lines) of manually delineated corpus callosum volumes (CCV). B. Bland-Altman plots showing intra-observer reproducibility and upper and lower 95\% Cls (dotted lines) of automated whole brain segmentations (whole brain volume (WBV)); LoA - limits of agreement.

while WBV values ranged from $695.1 \mathrm{~cm}^{3}$ to $1,364.3 \mathrm{~cm}^{3}$ (median: 1,157.8 $\mathrm{cm}^{3}$ ). Correlation between baseline CCV and WBV was not significant $(r=0.25, \mathrm{p}=0.37$; Fig. 2).

At follow-up, CCV values ranged from $4.2 \mathrm{~cm}^{3}$ to $9.5 \mathrm{~cm}^{3}$ (median: $7.5 \mathrm{~cm}^{3}$ ). Seven out of 23 patients (30.4\%) had a greater than $9 \%$ reduction in $\mathrm{CCV}$ at the $2^{\text {nd }}$ timepoint, meaning a real reduction beyond measurement variability (Fig. 3). At follow-up, WBV ranged from $718.0 \mathrm{~cm}^{3}$ to $1,400.7 \mathrm{~cm}^{3}$ (median: $1,144.0 \mathrm{~cm}^{3}$ ). Five out of $15 \mathrm{pa}$ tients $(33.3 \%$ ) had a greater than $1.2 \%$ reduction in WBV at the $2^{\text {nd }}$ timepoint, indicating a real reduction beyond measurement variability. 


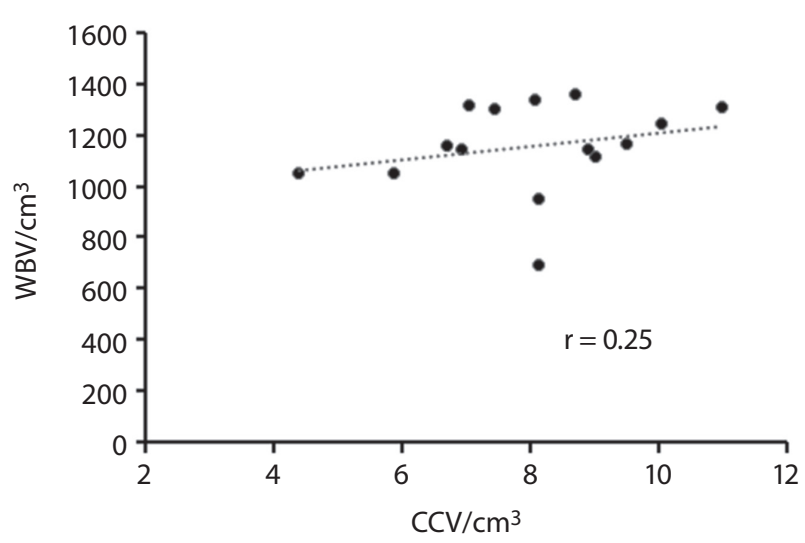

Fig. 2. Scatter plot showing the relationship between the measurements of corpus callosum volume (CCV) and whole brain volumes (WBV)

Five out of 7 patients who were recognized as having CCV loss at follow-up were not picked up by the WBV measurements. Similarly, 3 out of 5 patients identified as having WBV loss at follow-up were not picked up by the CCV measurements, confirming the poor correlation between the 2 assessments of brain parenchymal loss.

\section{Corpus callosum and WBV loss in low-vs high-dose radiation regimens}

Of the 7 patients with CCV loss greater than measurement variability, 2 were in group 1, 3 in group 2 and 2 in group 3. Of the 5 patients with WBV loss greater than measurement variability, 1 was in group 1, 2 in group 2 and 2 in group 3. The mean CCV loss was -5.1 in group 1, -6.9 in group 2 and -5.5 in group 3 , while for WBV the values were $-1.6,-0.8$ and -0.3 , respectively.

\section{Discussion}

To our knowledge, this study is the first to evaluate the impact of RT on brain parenchyma in children using 2 different techniques for quantifying brain volume. Our study showed that both CCV and WBV decrease after RT in up to a third of the children. Importantly, however, this study has shown that CCV does not correlate with WBV, so that significant reductions in these measurements were seen in different individuals. They may represent different ways of estimating brain parenchymal volume loss and should not be assumed to be interchangeable.

In this small cohort of patients, it was not possible to demonstrate the dose distribution of $\mathrm{RT}$ in relation to the $\mathrm{CC}$ to establish whether a greater dose was received by this structure in those patients demonstrating the profound effects of white matter loss within this structure.

The CCV has been used to quantify white matter loss in a variety of oncologic and non-oncologic applications. A reduced CC area was noted among children with adrenoleukodystrophy, an effect of demyelination. ${ }^{12}$ Similar results have been observed in patients with attention deficit hyperactivity disorder, and among those with Williams syndrome. ${ }^{13-15}$ In oncology, white matter damage was observed primarily in children with MDL (as it is the most common malignant solid tumor in children) and CCV was used to detect white matter loss. ${ }^{4,9}$ Palmer et al. described a decrease in the CCV in 35 MDL patients following craniospinal irradiation. ${ }^{9}$ Other reports on white matter volume also show that the volume of normal-appearing white matter decreases in children treated for MDL with craniospinal irradiation. ${ }^{4}$ Data from the present study

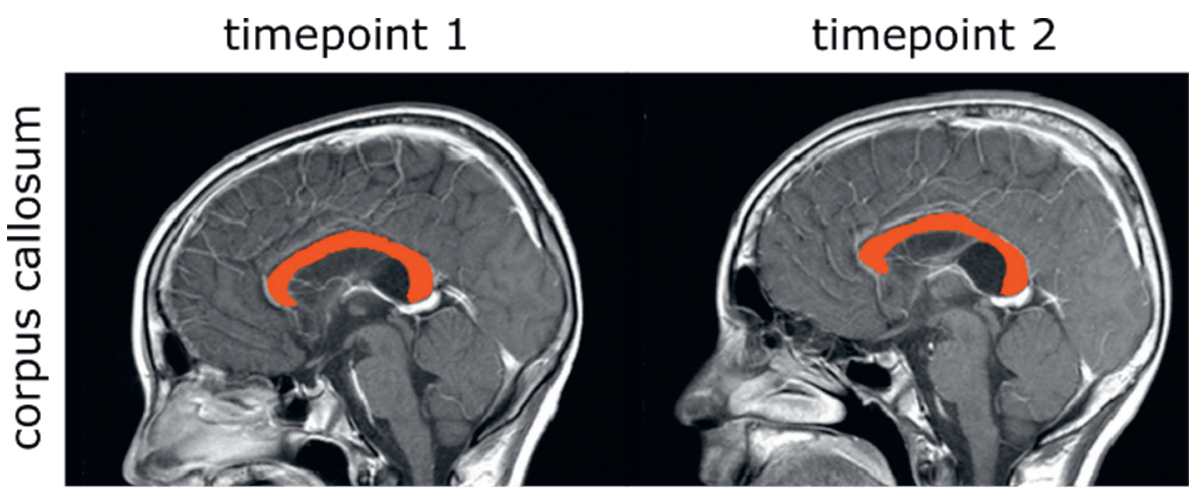

Fig. 3. Nine-year old patient with standard-risk medulloblastoma (SR MDL). Figures showing region of interest delineating the corpus callosum (CC) on a mid-sagittal T1W image (top row, A) and a mask segmenting the whole brain to derive a whole brain volume (WBV; bottom row, B) The cerebrospinal fluid (CSF) spaces are excluded in B

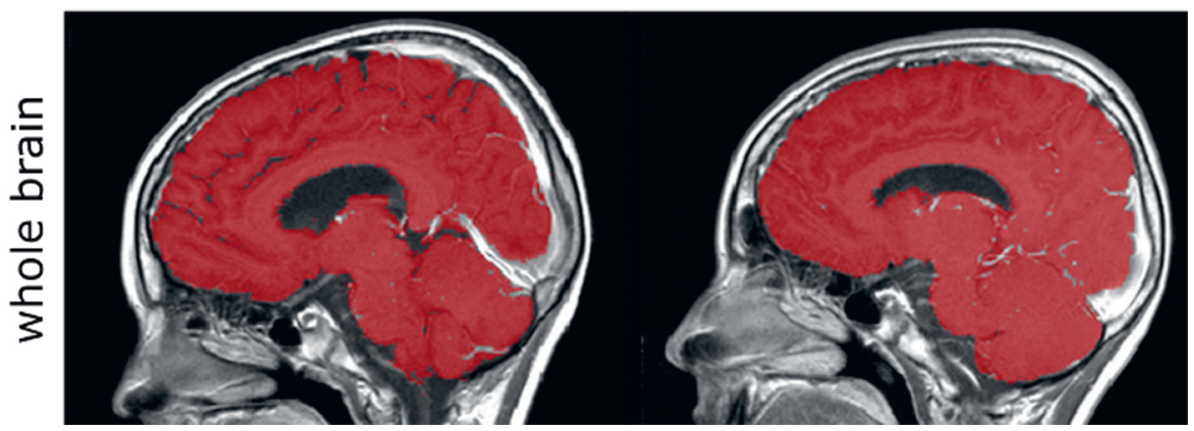


is consistent with these previous reports. The CCV measurements have also been used in children with primitive neuroectodermal tumors treated with high-dose thiotepa after hyperfractionated accelerated craniospinal radiotherapy (HART), revealing mild to severe neuroparenchymal volume loss following intensive sequential high-dose therapy with thiotepa given after HART regimen. ${ }^{5}$ This confirmed previous results from a similar study, but where a different method of estimating brain volume was used. ${ }^{6}$

Linear increases in white matter volumes, occurring during normal maturation, have been documented in a largescale study of 145 healthy individuals aged between 4 and 20 years. ${ }^{13}$ The size of the CC also increases with age into early adulthood: this has been demonstrated in a study of 109 healthy subjects aged 7-32 years. ${ }^{16,17}$ The growth of $\mathrm{CC}$ is regarded as the direct result of myelination of callosal axons present at birth. Therefore, a decline in CCV and WBV is opposite to what would be expected with normal maturation and is likely to be directly related to the effects of RT. In the timeframe of this study, where follow-up times were lengthy and patient growth and maturation would have occurred, it may well be that increases in CCV (and even in WBV) as a result of maturation would have masked any treatment-related reduction in CCV and WBV. Nevertheless, the 7 patients who showed CCV loss were followed up between 9 and 73 months (median: 21 months), while the 5 patients with WBV loss underwent follow-up imaging between 10 and 96 months (median: 60 months), indicating that follow-up time is not a primary confounding factor here.

Measurement error was greater for CCV than for WBV, but this is unsurprising as it depended on manual delineation rather than a computer-based automated process, albeit one that required manual correction. Manual segmentation is highly intensive and time-consuming, can be prone to errors, and may suffer from both inter- and intra-rater variability. ${ }^{18}$ In several studies, automation has improved the reliability of ROI delineation and hence of the derived measurements. ${ }^{19,20}$ Nevertheless, manual measurement of CCV enabled us to exclude artifacts from areas of surgical resection, which can potentially affect the assessments. However, an accurate outlining of the CC remains a challenge. One of the factors that may affect the actual accuracy of measurement is the impact of RT on CC irregularity of size and shape in relation to other brain structures. ${ }^{21-23}$ Another challenge for precisely outlining the CC may result from its decreased signal intensity as maturation of the axonal cytoskeleton occurs. Signal intensity of the CC decreases during childhood and adolescence, thereby reducing image contrast between the $\mathrm{CC}$ and surrounding brain parenchyma, which may have affected outlining in our cohort who were mainly above the age of $8 .^{16}$

The Freesurfer-based approach employed for WBV measurements was more robust than the approach for segmenting the $\mathrm{CC}$, as it reduced the potential for rater bias. The resulting whole-brain segmentations also included more tissue, minimizing the effect of any variability.
However, performing the measurements was laborious and time-consuming, as extensive manual correction of the masks was required. Even with semi-automated segmentation, the time required for each mask was around $3 \mathrm{~h}$. This meant that it was only feasible to perform the repeatability study in 5 cases, although ideally all patients should have been included.

There are several limitations of this study. Firstly, its retrospective nature meant that time after RT was variable (6-96 months), although we included only those patients in whom a reasonable time following treatment had elapsed (6 months). Nevertheless, this meant that we could not control neither for ongoing effects of RT nor for brain maturation that may have confounded the measurements. Secondly, the imaging was performed using a variety of protocols, which meant that differences in T1- and T2-weighting would have affected image contrast and the conspicuity of the structures being outlined, leading to measurement variability. To avoid these types of errors, we outlined sequences on the sagittal T1-W and repeated the measurement after 6 months to verify measurement repeatability. Because of the retrospective nature of this study, variations in protocols meant that imaging parameters such as in-plane resolution also varied between patients and timepoints. This variation in protocols, combined with a restricted field of view and variable image quality, resulted in the misclassification of tissue in the automated brain segmentation. As a result, extensive manual correction of the segmentations was required. In addition to this, the field of view used for the images resulted in the lateral portions of the brains being clipped. We compensated for the clipping by only considering brain regions that were included at both timepoints. Finally, because we only selected patients in whom paired, reasonably good quality imaging studies were available, our sample size was small and resulted in a low statistical power.

In summary, our study has confirmed a decline in both CCV and WBV values in around $1 / 3$ of the cases following cranial RT in children, but this decline was not related to radiation dose. However, this also suggests that both these measurements may not be used interchangeably, and may actually be sensitive to different factors. Both measurements, however, were relatively robust and even manually delineated CCV measurements would be expected to detect volume decreases of more than $9 \%$. The appropriate measurement method should be selected, and its variability established when using CCV or WBV assessments in clinical trials.

\section{ORCID iDs}

Elwira Szychot (D) https://orcid.org/0000-0002-1598-7018 Kiran K Seunarine (D) https://orcid.org/0000-0002-2467-7716 Carlos Andrés Robles (D) https://orcid.org/0000-0002-7256-0731 Henry Mandeville (D) https://orcid.org/0000-0001-6820-8578 Kshitij Mankad (D) https://orcid.org/0000-0001-5979-9337 Christopher Clark (D) https://orcid.org/0000-0002-8237-6065 Jarosław Peregud-Pogorzelski (i) https://orcid.org/0000-0003-2634-6002 Nandita deSouza (D) https://orcid.org/0000-0003-4232-476X 


\section{References}

1. Johnson KJ, Cullen J, Barnholtz-Sloan JS, et al. Childhood brain tumor epidemiology: A brain tumor epidemiology consortium review. Cancer Epidemiol Biomarkers Prev. 2014;23(12):2716-2736.

2. Greene-Schloesser D, Robbins ME, Peiffer AM, Shaw EG, Wheeler KT, Chan MD. Radiation-induced brain injury: A review. Front Oncol. 2012; 19(2):73.

3. Khong PL, Leung LH, Chan GC, et al. White matter anisotropy in childhood medulloblastoma survivors: Association with neurotoxicity risk factors. Radiology. 2005;236(2):647-652.

4. Reddick WE, Russell JM, Glass JO, et al. Subtle white matter volume differences in children treated for medulloblastoma with conventional or reduced dose craniospinal irradiation. Magn Reson Imaging. 2000;18(7):787-793.

5. Szychot E, Seunarine K, Mankad K, et al. Impact of induction chemotherapy, hyperfractionated accelerated radiotherapy and high-dose thiotepa on brain volume loss and functional status of children with primitive neuroectodermal tumour. Pediatr Blood Cancer. 2017;64(11). doi:10.1002/pbc.26619

6. Thust SC, Blanco E, Michalski AJ, et al. MRI abnormalities in children following sequential chemotherapy, hyperfractionated accelerated radiotherapy and high-dose thiotepa for high-risk primitive neuroectodermal tumours of the central nervous system. J Med Imaging Radiat Oncol. 2014;58(6):683-690.

7. Shan ZY, Liu JZ, Glass JO, Gajjar A, Li CS, Reddick WE. Quantitative morphologic evaluation of white matter in survivors of childhood medulloblastoma. Magn Reson Imaging. 2006;24(8):1015-1022.

8. Reddick WE, Shan ZY, Glass JO, et al. Smaller white-matter volumes are associated with larger deficits in attention and learning among long-term survivors of acute lymphoblastic leukemia. Cancer. 2006; 106(4):941-949.

9. Palmer SL, Reddick WE, Glass JO, Gajjar A, Goloubeva O, Mulhern RK Decline in corpus callosum volume among pediatric patients with medulloblastoma: Longitudinal MR imaging study. AJNR Am J Neuroradiol. 2002;23(7):1088-1094.

10. Jenkinson M, Beckmann CF, Behrens TE, Woolrich MW, Smith SM. FSL. Neuroimage. 2012;62(2):782-790.

11. Athinoula A. Martinos Center for Biomedical Imaging, Charlestown, MA, USA. http://surfer.nmr.mgh.harvard.edu. Accessed 27 November 2018).
12. Barkovich AJ. Concepts of myelin and myelination in neuroradiology. AJNR Am J Neuroradiol. 2000;21(6):1099-1109.

13. Giedd JN, Castellanos FX, Casey BJ, et al. Quantitative morphology of the corpus callosum in attention deficit hyperactivity disorder. Am J Psychiatry. 1994;151(5):665-669.

14. Baumgardner TL, Singer HS, Denckla MB, et al. Corpus callosum morphology in children with Tourette syndrome and attention deficit hyperactivity disorder. Neurology. 1996;47(2):477-482.

15. Schmitt JE, Eliez S, Warsofsky IS, Bellugi U, Reiss AL. Corpus callosum morphology of Williams syndrome: Relation to genetics and behavior. Dev Med Child Neurol. 2001;43(3):155-159.

16. Keshavan MS, Diwadkar VA, DeBellis M, et al. Development of the corpus callosum in childhood, adolescence and early adulthood. Life Sci. 2002;70(16):1909-1922.

17. Pujol J, Vendrell $P$, Junqué C, Martí-Vilalta JL, Capdevila A. When does human brain development end? Evidence of corpus callosum growth up to adulthood. Ann Neurol. 1993;34(1):71-75.

18. Despotović I, Goossens B, Philips W. MRI segmentation of the human brain: Challenges, methods, and applications. Comput Math Methods Med. 2015;2015:450341.

19. Ashton EA, Takahashi C, Berg MJ, Goodman A, Totterman S, Ekholm S. Accuracy and reproducibility of manual and semi-automated quantification of MS lesions by MRI. J Magn Reson Imaging. 2003;17(3): 300-308.

20. Rosenbluth $\mathrm{KH}$, Gimenez F, Kells AP, et al. Automated segmentation tool for brain infusions. PLoS One. 2013;8(6):e64452.

21. Wang D, Shi L, Chu WC, Paus T, Cheng JCY, Heng PA. A comparison of morphometric techniques for studying the shape of the corpus callosum in adolescent idiopathic scoliosis. Neuroimage. 2009;45(3): 738-748.

22. Collinson SL, Gan SC, Woon PS, et al. Corpus callosum morphology in first-episode and chronic schizophrenia: Combined magnetic resonance and diffusion tensor imaging study of Chinese Singaporean patients. Br J Psychiatry. 2014;204(1):55-60.

23. Pekala JS, Mamourian AC, Wishart HA, Hickey WF, Raque JD. Focal lesion in the splenium of the corpus callosum on FLAIR MR images: A common finding with aging and after brain radiation therapy. AJNR Am J Neuroradiol. 2003;24(5):855-861. 